\title{
High degree of non-genetic phenotypic variation in the vascular system of crayfish: a discussion of possible causes and implications
}

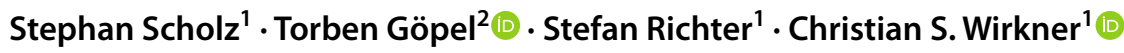

Received: 20 November 2020 / Revised: 6 July 2021 / Accepted: 25 July 2021 / Published online: 12 August 2021

(c) The Author(s) 2021

\begin{abstract}
In this study, the hemolymph vascular system (HVS) in two cambarid crayfishes, i.e. the Marbled Crayfish, Procambarus virginalis Lyko, 2017 and the Spiny Cheek Crayfish, Faxonius limosus (Rafinesque, 1817), is investigated in regard of areas of non-genetic phenotypic variation. Despite their genetic identity, specimens of $P$. virginalis show variability in certain features of the HVS. Thus, we describe varying branching patterns, sporadic anastomoses, and different symmetry states in the vascular system of the marbled crayfish. We visualize our findings by application of classical and modern morphological methods, e.g. injection of casting resin, micro-computed tomography and scanning electron microscopy. By comparing our findings for $P$. virginalis to the vasculature in sexually reproducing crayfishes, i.e. F. limosus and Astacus astacus, we discuss phenotypic variation of the HVS in arthropods in general. We conclude that constant features of the HVS are hereditary, whereas varying states identified by study of the clonal $P$. virginalis must be caused by non-genetic factors and, that congruent variations in sexually reproducing $F$. limosus and A. astacus are likely also non-genetic phenotypic variations. Both common causal factors for non-genetic phenotypic variation, i.e., phenotypic plasticity and stochastic developmental variation are discussed along our findings regarding the vascular systems. Further aspects, such as the significance of nongenetic phenotypic variation for phylogenetic interpretations are discussed.
\end{abstract}

Keywords Evolutionary morphology $\cdot$ Phenotypic plasticity $\cdot$ Circulatory system $\cdot$ Heart $\cdot$ Artery

\section{Introduction}

\section{Intraspecific variability of arthropod circulatory systems}

The circulatory systems of arthropods have been shown to be subject to a high degree of intraspecific variability. Such intraspecific variability can be found all across the arthropod tree of life and within all constituents of the hemolymph circulatory system. In Xiphosura, various prominent regions of both, the vascular system and the lacunar system have been shown to vary intraspecifically, as well as even the number of ostia (Göpel and Wirkner 2015). In arachnids, a significant

Christian S. Wirkner

christian.wirkner@uni-rostock.de

1 Institut für Biowissenschaften, Universität Rostock, Allgemeine und Spezielle Zoologie, Universitätsplatz 2, 18055 Rostock, Germany

2 Department of Biological Sciences, University of North Texas, 1155 Union Circle \#305220, Denton, TX 76203, USA degree of intraspecific variability was found especially in the supply of the prosomal ganglion and adjacent structures (Huckstorf et al. 2015; Runge and Wirkner 2016). The same can be reported for the vascular supply in at least scutigeromorph chilopods (unpublished data). Within crustaceans, the Decapoda so far have been studied most extensively in regard to intraspecific variability of vascular morphology (Baumann 1921; Imafuku 1993; Vogt et al. 2009; Keiler et al. 2013, 2015, 2016). Most prominently, the descending artery, one of the major cardiac arteries and responsible for the supply of the entire ventral cephalothorax, can be either paired or unpaired passing the gut on either its right or left side (Imafuku 1993; Vogt et al. 2009). The relative frequency of the different morphological conditions (i.e. right-sided, left-sided, or symmetrical descending artery), appears to differ greatly between decapod taxa and in taxa with asymmetric habitus, often is independent from handedness (Imafuku 1993). While the question of whether such intraspecific variability is genetically determined could not be answered in other species, the parthenogenetic marbled crayfish showed the same variability in its genetically identical offspring (Vogt et al. 2009; Gutekunst 
et al. 2018). These findings point towards non-genetic phenotypic variation as cause for this kind of variability (Vogt et al. 2009).

\section{Phenotypic plasticity and stochastic developmental variation}

Phenotypic plasticity generally refers to different phenotypes (i.e. different morphological, physiological and/or behavioral traits) of the same genotype in response to changing environmental conditions to which the organism is exposed (West-Eberhard 1989; Kelly et al. 2012; Pelster and Burggren 2018). Phenotypic plasticity can be induced for instance by abiotic environmental factors (e.g. temperature) or by presence of other organisms such as predators, e.g. in water fleas (mediated through chemical cues; Tollrian 1993, 1995). As direct response to environmental factors, phenotypic plasticity is more frequent and thus considered advantageous in changing environments (Langerhans and DeWitt 2002; Ghalambor et al. 2007; Kelly et al. 2012; Chevin and Hoffmann 2017; Pelster and Burggren 2018).

Another aspect of non-genetic phenotypic variation is stochastic developmental variation (Vogt 2015, 2017, 2020; Angers et al. 2020). Although subject to the same environmental conditions, genetically identical organisms can develop different phenotypes due to stochastic alterations of the epigenome (Leung et al. 2016; Atlasi and Stunnenberg 2017; Angers et al. 2020; Vogt 2020). Thus, stochastic developmental variation, unlike phenotypic plasticity, is no direct response to specific change of environmental factors but considered a bet-hedging strategy (Simons 2014; Furness et al. 2015; Vogt 2015; Leung et al. 2016; Angers et al. 2020).

Environmentally induced phenotypic plasticity and stochastic developmental variation, however, are no mutually exclusive strategies but complementary sources of phenotypic variation (Vogt 2020). While phenotypic plasticity is considered advantageous in habitats with rather predictable environmentally changes, stochastic developmental variation is considered a diversifying bet-hedging strategy suited for less predictable or unpredictable environments (Leung et al. 2016). In natural environments, which cannot be characterized binarily as either predictable or unpredictable, the coexistence of phenotypic plasticity and stochastic developmental variation is likely to be a beneficial compromise for environments with both predictable and unpredictable changes of conditions (Leung et al. 2016).

\section{The marbled crayfish as 'model organism' for phenotypic variation}

The parthenogenetic reproduction makes Procambarus virginalis unique among the decapod crustaceans (Martin et al.
2007; Vogt et al. 2008) and interesting as object of research. The marbled crayfish $P$. virginalis first appeared in German aquarium trade in the mid-1990s (Martin et al. 2010). Due to its exclusively parthenogenetic reproduction with only females present it soon gained the interest of researchers (Scholtz et al. 2003; Martin et al. 2007). After it had been shown that $P$. virginalis is a triploid form of the North American crayfish Procambarus fallax (Hagen, 1870) (Martin et al. 2016; Gutekunst et al. 2018), the marbled crayfish has recently been described as separate species by Lyko (2017). The mode of parthenogenesis in $P$. virginalis is exclusively apomictic. In this kind of parthenogenesis, in which meiosis is suppressed and diploid (or in this case triploid) eggs are produced by mitosis only (Simon et al. 2003), all offspring is both genetically identical among themselves and to the mother (Martin et al. 2007; Gutekunst et al. 2018). Furthermore, $P$. virginalis has a relatively short generation time (compared to other decapods), features high robustness regarding rearing conditions and thus is well suited for the study of both phenotypic plasticity and stochastic developmental variation (Vogt 2008, 2020; Vogt et al. 2008).

In the case of $P$. virginalis, Vogt et al. (2008) observed phenotypical variation of life history traits, fitness, pigmentation as well as dispersion of setae and antennal aesthetascs.

Previously, we recorded the varying symmetry states of descending artery as an example of stochastic developmental variation (Vogt et al. 2009). More recently, we have described the gross morphology of the HVS of $P$. virginalis based on its constant patterns (Scholz et al. 2018), whereas in in the present work, we investigate the hemolymph vascular system of the marbled crayfish in regard to varying patterns of the vascular system as the result of non-genetic variation.

We describe different kinds of intraspecific variability of vascular morphology exemplified by visualizations of alternative vascular morphologies in several individuals of $P$. virginalis. By comparing these data with that of the two sexually reproducing species Faxonius limosus (Rafinesque, 1817) (own investigation) and Astacus astacus (Linnaeus, 1758) (data from the literature; Baumann 1921), putative variable patterns in vascular morphology in crayfish in general are identified. As a starting point for the investigation of stochastic developmental variation (as well as phenotypic plasticity) of internal morphology in decapod circulatory systems, this phenomenon is discussed in the framework of evolutionary morphology.

\section{Materials and methods}

\section{Species studied}

Procambarus virginalis Lyko, 2017 and Faxonius limosus (Rafinesque, 1817) 
Adult specimens of $P$. virginalis were bought at a pet shop, and reared individually in 6-L aquaria filled with tap water at room temperature $\left(22 \pm 3{ }^{\circ} \mathrm{C}\right) ; 48$ specimens were studied. For histology, one juvenile (3 $\mathrm{mm}$ carapace length) as well as twelve recently hatched animals were dissected. For injection preparations, specimens with a carapace length of at least $1 \mathrm{~cm}$ were used. Adult specimens of $F$. limosus with a carapace length of about $4.5 \mathrm{~cm}$ were caught from a lake near Rostock (Hohensprenzer See) in November 2009. These animals were kept together in a $300-\mathrm{L}$ aquarium filled with tap water at $5{ }^{\circ} \mathrm{C}$ and equipped with shelters; 22 specimens were studied. All animals at any life stage were fed ad libitum with "Novo Crabs" food chips (JBL, Neuhofen, Germany).

\section{Histology}

The specimens were fixed in Bouin's fixative and dehydrated in a graded ethanol series. After an intermediate washing step in acetone, the dissected parts were embedded in araldite epoxy resin. Series of semi-thin sections $(1 \mu \mathrm{m})$ were made with a rotary microtome (Leica, RM 2165) using either glass or diamond knifes. Sections were stained with a mixture of $1 \%$ toluidine blue, $1 \%$ sodium-tetraborate and $1 \%$ pyronin $\mathrm{G}$ in an aqueous solution for about $35 \mathrm{~s}$ at $60{ }^{\circ} \mathrm{C}$.

\section{Micro-CT}

$\mathrm{X}$-ray imaging was performed with a phoenix|x-ray nanotom ${ }^{\circledR}$ high resolution micro-computed tomography system (GE Sensing and Inspection Technologies, Wunstorf, Germany) using the proprietary software datos. $\mathrm{X}$ acquisition and datos.x reconstruction. Image stacks of virtual sections were exported with the software VG Studio max (Volume Graphics GmbH, Heidelberg, Germany). Some scans were performed using the X-ray Microscope Xradia 410 Versa (Carl Zeiss X-Ray Microscopy, Pleasanton, CA, USA).

\section{D reconstruction}

Every fifth semi-thin section of the series was photographed with a Zeiss AXIO Imager.M1 microscope equipped with a Zeiss AXIOCAM ICc3 camera (Carl Zeiss Microscopy $\mathrm{GmbH}$, Jena, Germany). Digitized sections were aligned automatically and corrected manually with the software Autoaligner 6.0.1. (Bitplane AG, Zurich, Switzerland). Visualizations of both the digitized section and the micro-CT data were performed with the software Imaris (version 6.4. and 7.0, Bitplane AG, Zurich, Switzerland), and Amira 6.4 (Thermo Fisher Scientific, Waltham, MA, USA).

\section{Resin injection}

For in-situ vessel depiction and following preparation, casting resins were injected into the heart of specimens of both species. Three different casting resins were applied: (1) PU4ii (vasQtec, Zurich, Switzerland), based on polyurethane, (2) Mercox II (Ladd Research Industries Inc, Williston, VT, USA), and (3) Mercox CL- 2R-5 (SPI-Suplies, West Chester, PA, USA), both based on methyl methacrylate. $F$. limosus specimens were injected with PU4ii while all three resins were used for $P$. virginalis specimens. After curing of the resins, different specimens were either fixed in Bouin's fixative (see "Histology") for the use in micro$\mathrm{CT}$ or dissolved as corrosion casts following the procedure described in Wirkner and Richter (2004) for scanning electron microscopy.

\section{Scanning electron microscopy}

Corrosion casts were sputter coated with gold using a BalTec SCD 004 (duration: 100 s; at the Electron Microscopy Center of the Universität Rostock) and an Emitech, K500 (duration: $100 \mathrm{~s}$; at the Zoological Insitute of the FSU Jena). Scanning electron micrographs were taken with FEI Company, XL30 ESEM TMP (at the FSU Jena) and a Zeiss, DSM 960A (at the Universität Rostock).

\section{Image management and processing}

Irfan-View 4.0 software was used to convert batches of the digitized images into grayscale, to invert and rename them. CorelDraw Graphics Suite X3 was used to create schematic depictions and to arrange the figure plates.

\section{Terminology}

The terminology used in this work is based on the Ontology of the Arthropod Circulatory System (Wirkner et al. 2017). All parts of the circulatory systems are considered morphemes (Richter and Wirkner 2014), i.e. any reference to homology and/or function are explicitly excluded from any of the given descriptions.

\section{Results}

In the vascular morphology of $P$. virginalis and $F$. limosus, we found several areas showing intraspecific variability of three different kinds: (1) variability in branching patterns of arterial systems, (2) variable occurrence of anastomoses, and (3) variable asymmetries (Fig. 2). In the following, these three kinds of variability will be exemplified. The gross morphology of the vascular system of both species has been 
described in Scholz et al. (2018) and is also summarized in Fig. 1.

\section{Variability in branching patterns}

Some artery systems show intraspecific variability in the branching pattern of arteries. This includes different sequence of off-branchings as well as common origins of arteries which are separate in other specimens. In the following, two artery systems, which are subject to a high degree of intraspecific variability in both species, will be described to exemplify this kind of variability (Fig. 2).

\section{Rostro-gastral vasculature}

The rostro-gastral vasculature is part of the anterior lateral artery system and supplies the rostral region as well as the stomach. This artery system consists of five main branches (rostral artery, epidermal artery, dorsal artery of the anterior gastric muscle, ventral artery of the anterior gastric muscle, and anterior gastric artery; Fig. 3A). In both crayfish species, the branching pattern is highly variable between the respective specimens and different patterns can be recognized (Fig. 3). In each species, there is a predominant pattern but even in the limited set of specimens investigated in this study ( $P$. virginalis: 14 specimens; F. limosus: 24 specimens), several alternatives could be identified (Fig. 3B, C). Although the same five main branches constitute this artery system in both crayfish species, only one of the alternative branching patterns could be identified in both species (Fig. 3B, C).

\section{Posterior aorta system}

In both investigated species, the posterior aorta system shows intraspecific variability, for instance in the branching pattern of its distal portion. The terminal bifurcation and the origins of the 4th to 6th posterior lateral arteries show significantly different conditions in different specimens (Fig. 4). The most frequent pattern is the "regular" pattern, with the 4th and 5th posterior lateral arteries emanating separately from the posterior aorta which ultimately bifurcates into the 6th posterior lateral arteries (Fig. 4A). Alternative patterns include a common origin of 5th and 6th posterior lateral arteries (Fig. 4B), a common origin of the 4th and 5th posterior lateral artery on one (Fig. 4C) or both sides (Fig. 4D), asymmetric common origins of two subsequent posterior lateral arteries (Fig. 4E), and the bifurcation of the posterior aorta anterior to the 4th posterior lateral artery (Fig. 4F). In addition to the alternative branching pattern, intersegmental anastomoses (Fig. 4, isan) occur in some specimens (for more on anastomoses, see below).
Fig. 1 Schematic overview of the hemolymph vascular system in the marbled crayfish $P$. virginalis (after Scholz et al. 2018). aao anterior aorta, ala anterior lateral arteries, $a r b$ : antenno-renal branch, $b a$ brain artery, cda caudal artery, chpa cardiac hepatic arteries, dsca descending artery, gib gastrointestinal branch, hea heart, $l b$ lateral branch of the anterior lateral artery, $m d b$ mandibular branch, $o a$ optic artery, $o a b$ occulo-antennular branch, os ostia of the heart, pao posterior aorta, pgal 1st posterior gastric artery, plp 3 artery of the 3rd pleopod, ppa 2 artery of the 2 nd peraiopod, $r g b$ rostro gastral branch, sta sterna artery, $t a$ telson artery, upd uropodal arteries

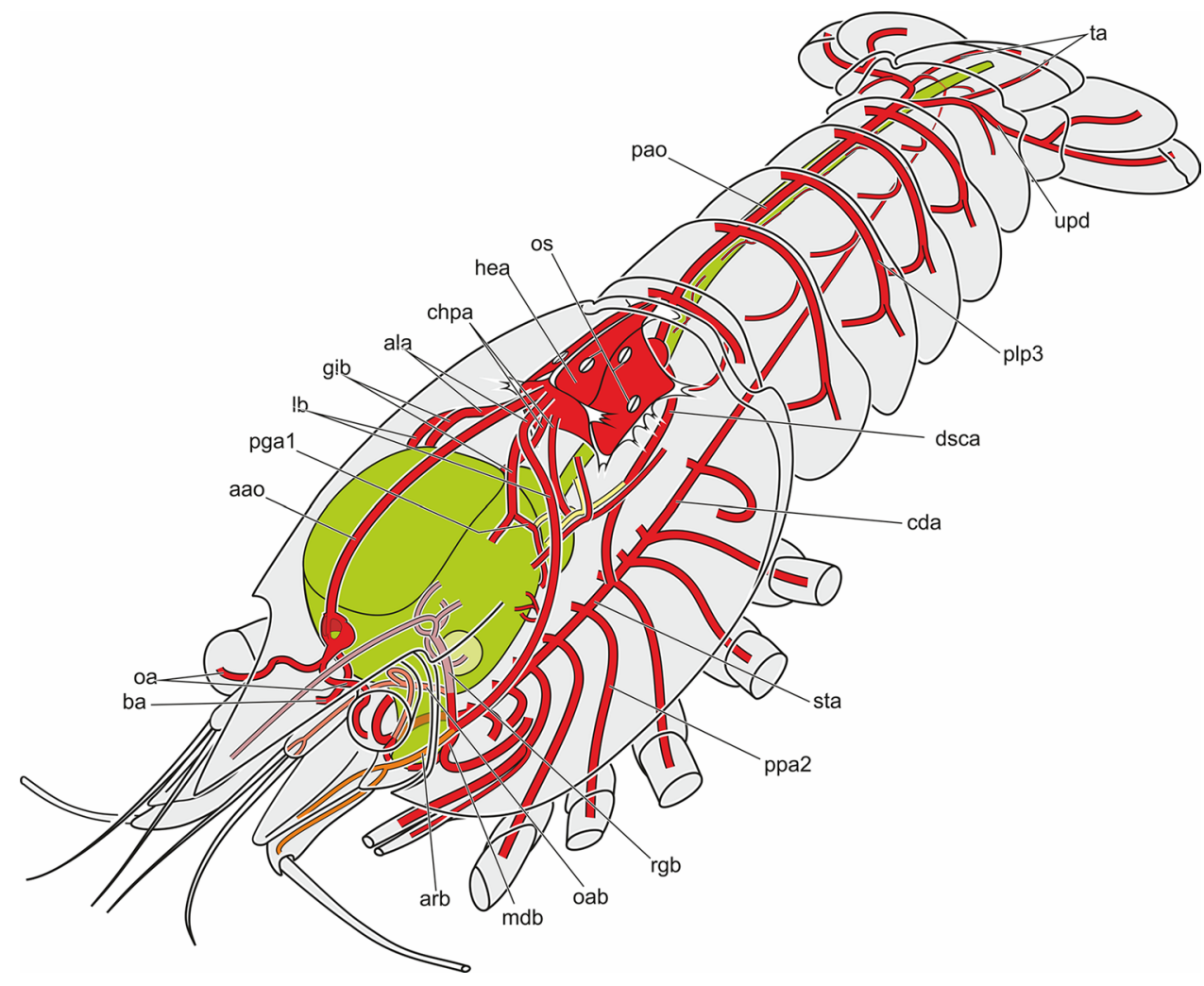



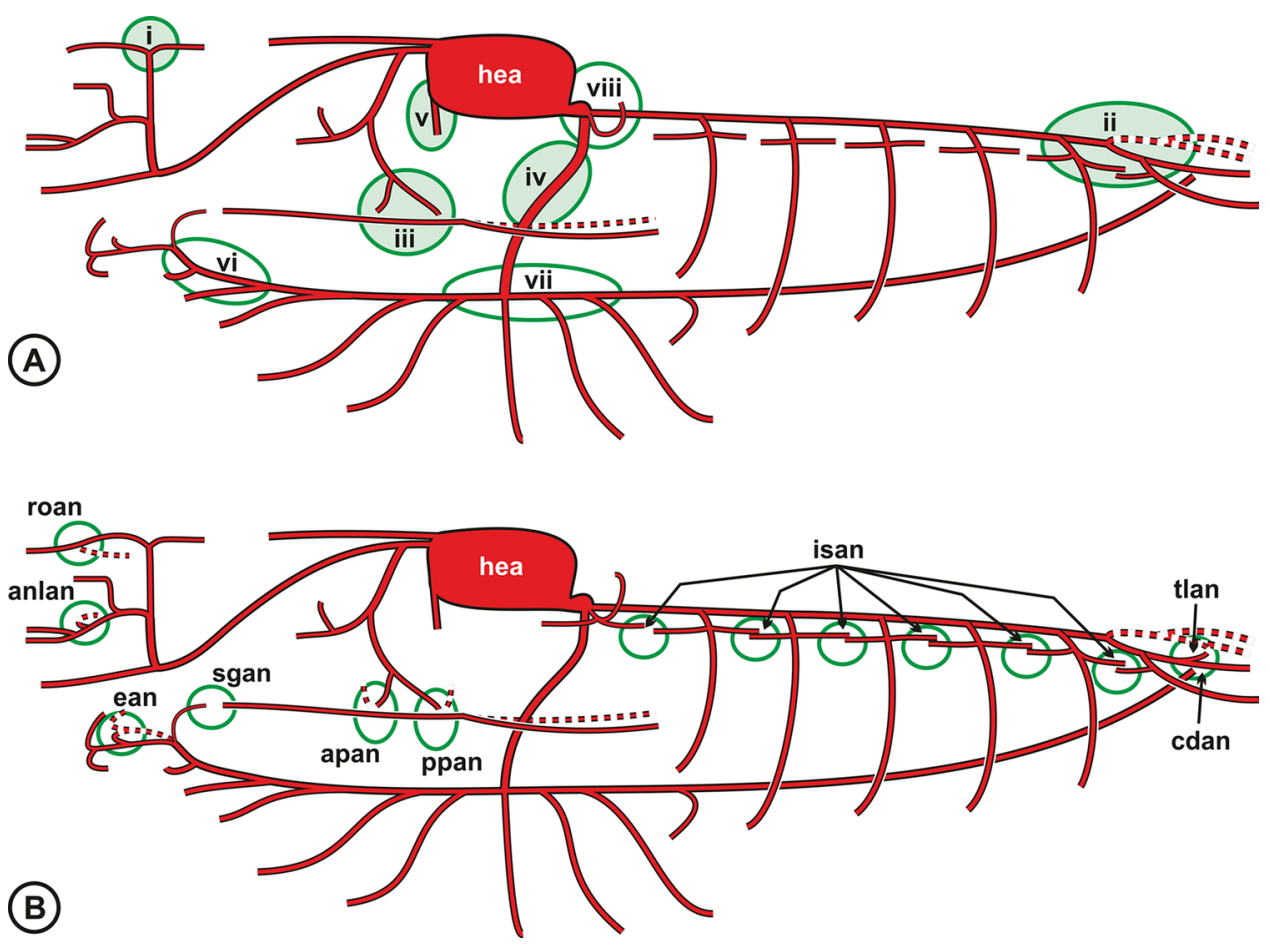

Fig. 2 Schematic overview of regions of the hemolymph vascular system showing intraspecific variability (lateral view). A Regions of the HVS showing variable branching patterns; filled circles show regions that are explained in the text. i: rostro-gastral vasculature; ii: terminal bifurcation of the posterior aorta; iii: branching pattern of the posterior gastric artery system; iv: course, number and caliber of the descending arteries; v: number of cardiac hepatic arteries; vi: junction pattern of arteries of the mouthparts in the anterior part of the ventral vessel system; vii: junction pattern of thoracopodal arteries in the ventral vessel system; viii: aortic bulb region. B Regions of anastomoses with intraspecific variability; anlan antennular anastomosis, apan anterior pyloric anastomosis, cdan caudal anastomosis, ean esophageal anastomosis, isan intersegmental anastomosis between neighboring posterior lateral arteries, roan rostral anastomosis, sgan subgastric anastomosis, tlan telsonic anastomosis between uropodal arteries

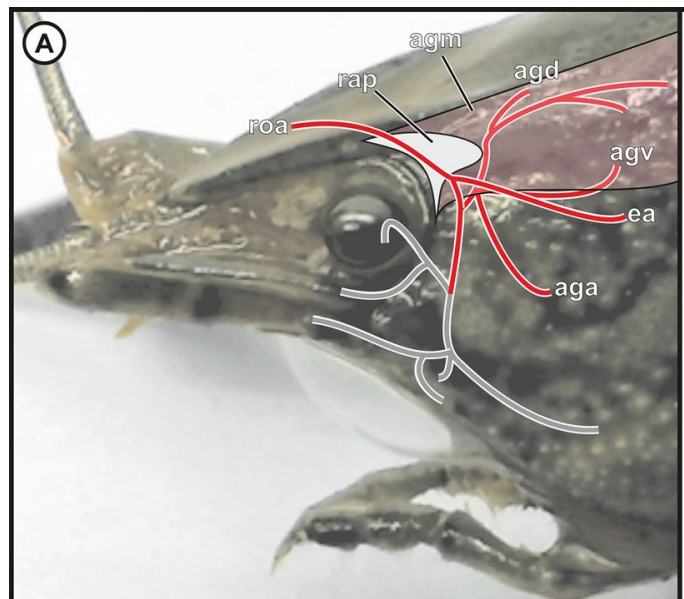

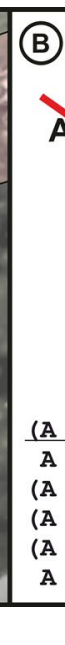

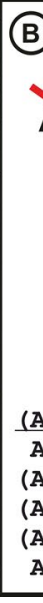

Fig. 3 Schematic illustration of the rostro-gastral vasculature. A Overview, from lateral, of the distal branching pattern in relation to the rostral apodeme (rap) and the anterior gastric muscle (agm) in $P$. virginalis. The five main arteries, i.e., rostral artery (roa=A), epidermal artery $(e a=B)$, dorsal artery of the anterior gastric muscle $(\mathrm{agd}=\mathrm{C})$ and ventral artery of the same $(\mathrm{agv}=\mathrm{D})$ and the anterior gastric artery $($ aga $=E)$. B - C Schemes in lateral view of the most frequent branching patterns. The intraspecifically varying regions are marked in black. B Pattern in P. virginalis and $\mathbf{C}$ in F. limosus. Below the drawings, the observed branching formulas are listed. The underline marks the only pattern occurring in both species. Brackets indicate arteries emanating from a common branch 
Fig. 4 Schematic illustration of the alternative branching patterns of the distal portion of the posterior aorta system. isan: intersegmental anastomosis; pao: posterior aorta; pla4-6: 4th to 6 th posterior lateral artery

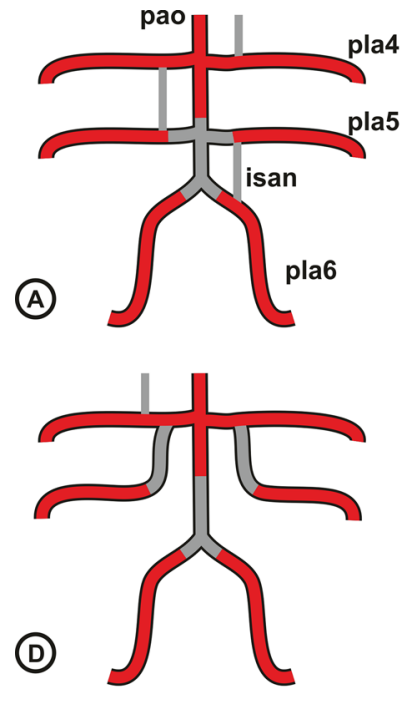

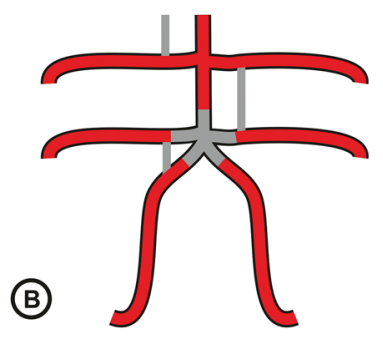
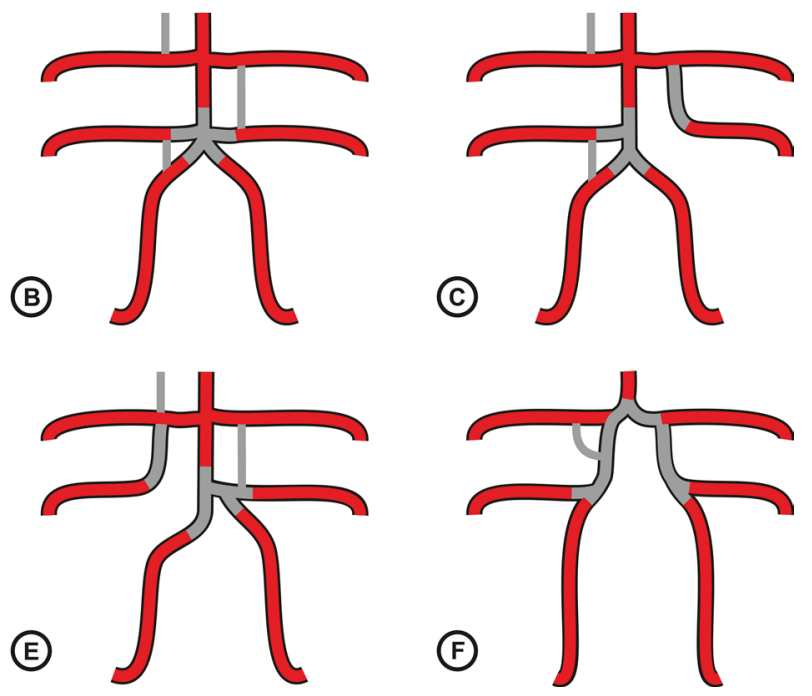

\section{Anastomoses}

Anastomoses, i.e. connecting arteries bypassing two artery systems, occur in numerous regions of the hemolymph vascular system in both crayfish species (Fig. 2B). These anastomoses, however, are not present in each single specimen but the occurrence rather shows remarkable intraspecific variability. In the following, variably occurring anastomoses will be described for exemplar regions of the crayfish vascular systems.

\section{Gastro-intestinal artery system}

The gastro-intestinal artery is part of the anterior lateral artery system. It runs medially and ramifies to form the gastro-intestinal artery system which supplies the stomach and the anterior midgut. The gastro-intestinal artery system of each side consists of three main branches, the three posterior gastric arteries (Fig. 5, pga1-3). In the ventral median line, right beneath the stomach, the subgastric artery runs longitudinally and is connected to the first posterior gastric artery via one or more anastomoses. In the marbled crayfish, the subgastric artery anteriorly sends off an anastomosis to connect with the sternal artery (Fig. 5A). In both species, intraspecific variability shows in the irregular occurrence of anastomoses between the anterior and posterior branch of the 1st posterior gastric artery of either side and the subgastric artery (Fig. 5B, C).

\section{Posterior aorta system}

As mentioned above, the posterior aorta system not only shows intraspecific variability in the branching pattern of its distal region but also in the occurrence of intersegmental anastomoses (Figs. 2, 3). These anastomoses connect the

posterior lateral arteries of neighboring pleomeres and can occur independently on both sides (Fig. 4). Between both extremes, i.e. no intersegmental anastomoses on the one hand and intersegmental anastomoses between all posterior lateral arteries forming two continuous parallels to the posterior aorta on the other hand, all graduations might occur as numerous patterns of intersegmental anastomoses were observed in both species.

\section{Variable asymmetries}

In the crayfish circulatory systems, asymmetries in vascular morphology are a frequent phenomenon. While some such asymmetries are the result of variable branching patterns (see above; Fig. 4), other asymmetries are based on the unilateral occurrence of entire artery systems or variable unilateral development.

\section{Descending artery}

The descending artery of $P$. virginalis is an unpaired artery in most specimens (see Table 1 for numbers in both species).

This artery passes the midgut either on the right or left side (as already described by Vogt et al. 2009). In some specimens, however, the descending artery is paired. In this case, both descending arteries can either be equally strong or one of the two arteries is stronger.

\section{Hepatic artery system}

Another remarkable case of asymmetry was found in the hepatic artery system of $P$. virginalis. A single cast of 25 observable ones of the hepatic artery showed only the left hepatic artery present supplying the entire midgut gland. In this specimen, the left hepatic artery gives rise to a 
Fig. 5 Schematic illustration of anastomoses of the gastrointestinal artery system (pga1). A Overview of the situation in lateral view, casts of 17 specimens of both, $P$. virginalis and $F$. limosus could be observed. The posterior gastric artery system with its three main branches (pga1-3) and the gastric mill (green) with the esophagus (es), gastrolith (gl) and pylorus (pyl). The posteriormost side branch (pga1a) and the subgastric artery (sga) supply the midgut gland via auxiliary hepatic vessels (ahv). The anastomosis between sternal artery (sta) and sga via median esophageal vessel (mev) was only found in P. virginalis; $\mathbf{B}-\mathbf{H}$ Schematic representations of the variations of the branching pattern found in the observed species, depicted in a ventral view; black are varying parts, grey is the sga in variations occurring in both species because its anterior section is absent in F. limosus; $\mathbf{B}$ Anastomosis of pgalb anterior from the pylorus is the most common variation in $P$. virginalis $(n=7)$; D Most common variation in $F$. limosus
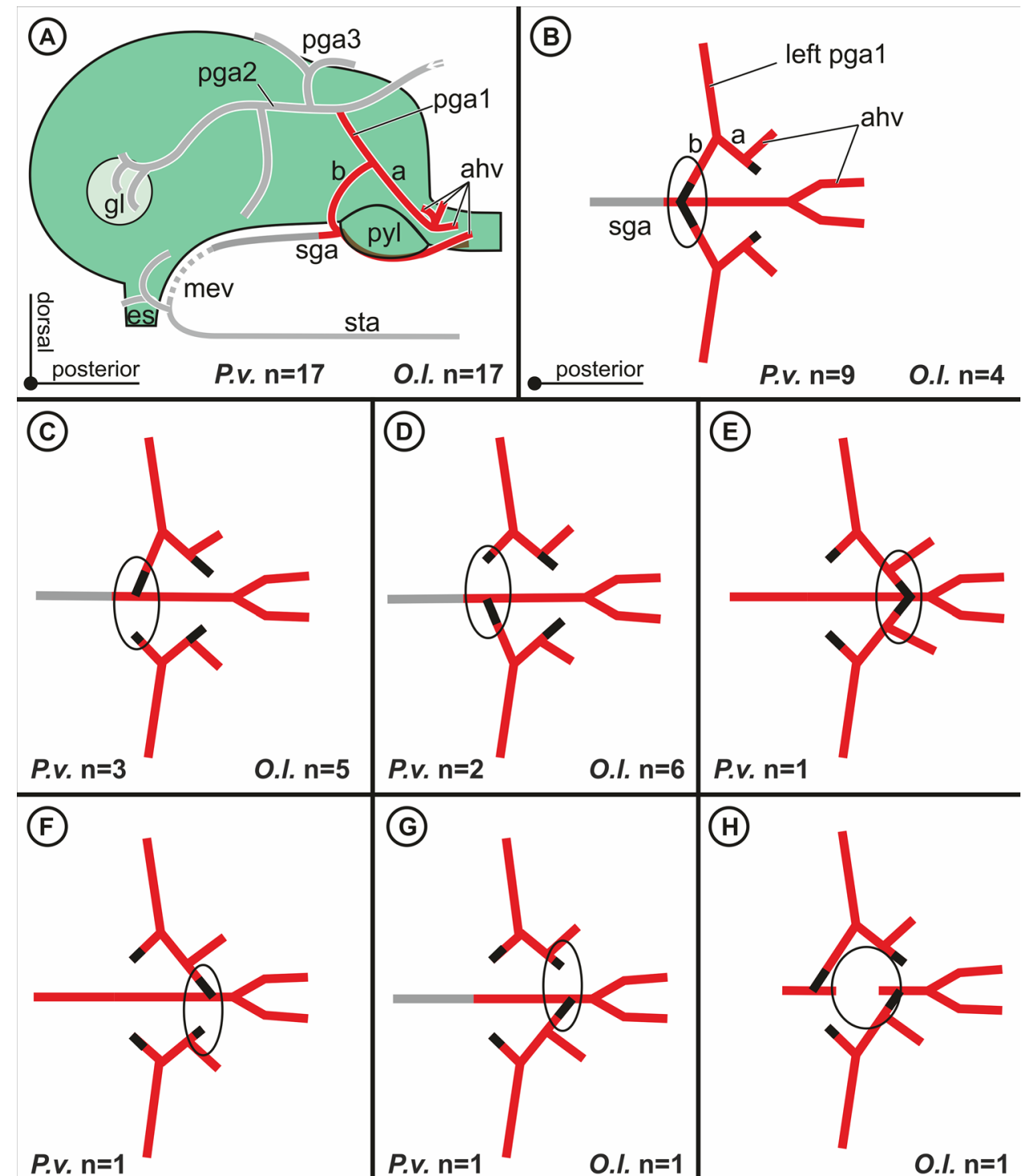

P.v. $\mathrm{n}=1$

P.v. $\mathrm{n}=1$

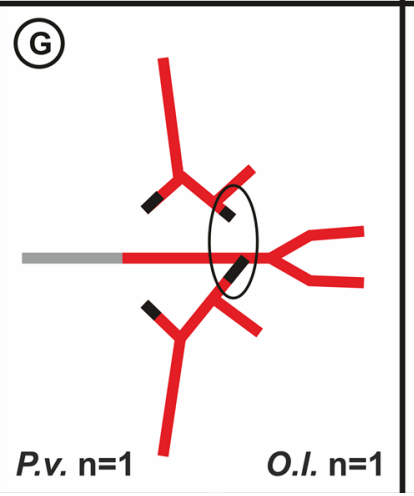

$\oplus$

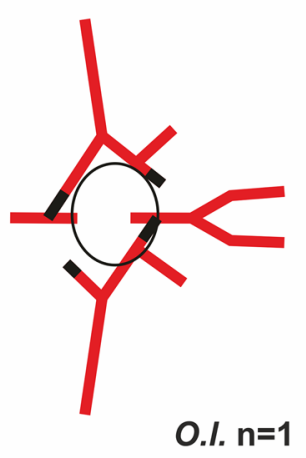

prominent branch crossing ventrally of the midgut to the right side, where it runs dorsally and ramifies into an elaborate vasculature supplying the entire right side of the midgut gland (Fig. 6).

\section{Discussion}

Although the development of arteries in crustaceans is not readily understood (Wirkner et al. 2013), intraspecific variability has also been reported for other arthropods such as other decapods (e.g. Keiler et al. 2013), stomatopods (unpublished data), horseshoe crabs (Göpel and Wirkner 2015), various arachnids (e.g. Klussmann-Fricke et al. 2012; Runge and Wirkner 2016; Huckstorf and Wirkner 2019) and chilopods (unpublished data). Our data show that distinct parts of the vascular morphology of crayfish are subject to non-genetic phenotypic variation.

In general, intraspecific variability in vascular morphology described for $P$. virginalis can either be ascribed to phenotypic plasticity or stochastic developmental variation due to their genetic identity (Martin et al. 2007; Gutekunst et al. 2018). Since all individuals of $P$. virginalis were kept under identical conditions, phenotypic plasticity induced by external environmental factors appears unlikely at first. Thus, a possible explanation for this non-genetic phenotypic variation is stochastic developmental variation as result of stochastic alterations of the epigenome (Vogt 2015, 2020). We were able to find congruent phenomena of intraspecific variability in the sexually reproducing crayfish $F$. limosus. Furthermore, Baumann (1921) described the same phenomena of intraspecific variability for Astacus astacus. Due to this congruence in variable patterns across all three species 


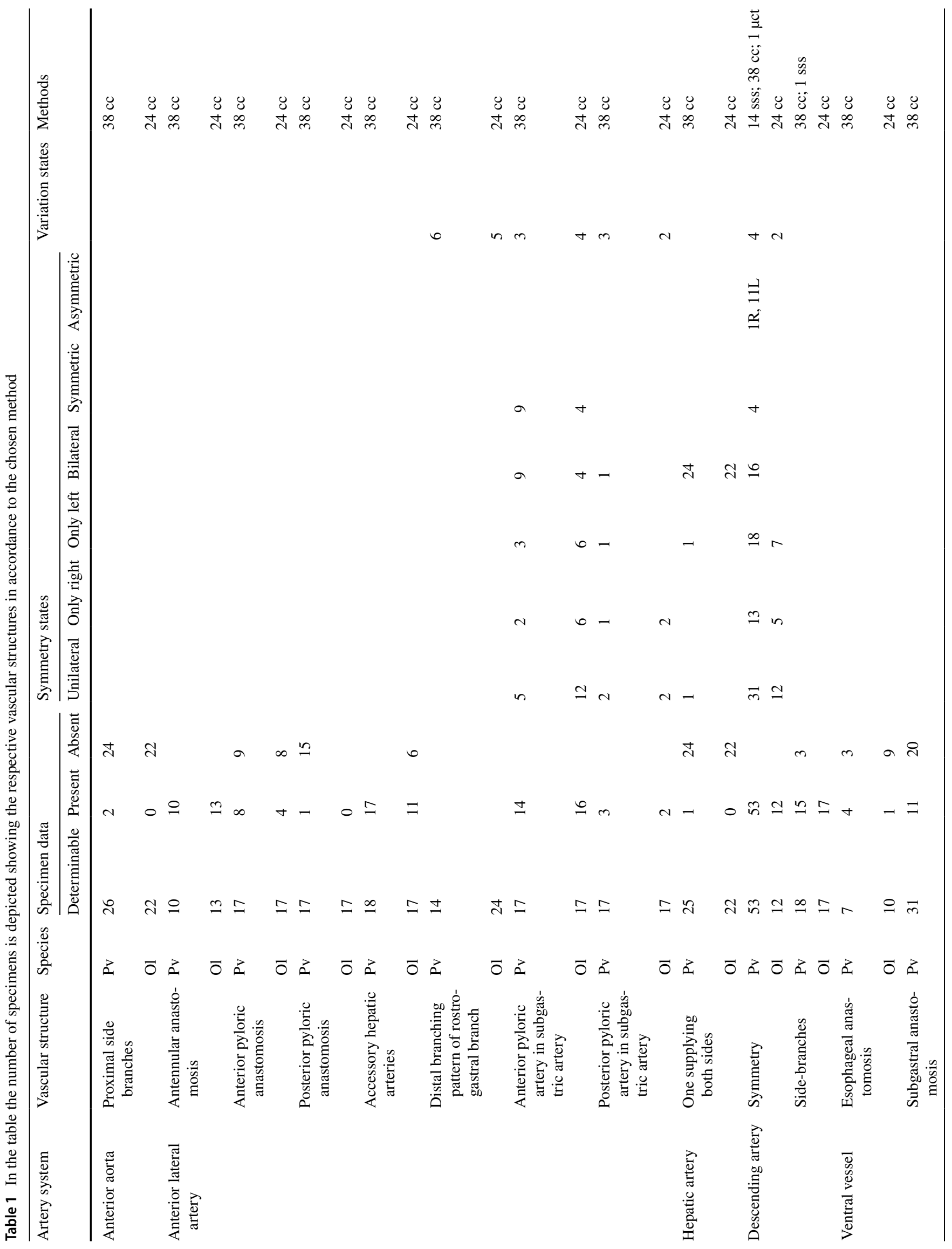




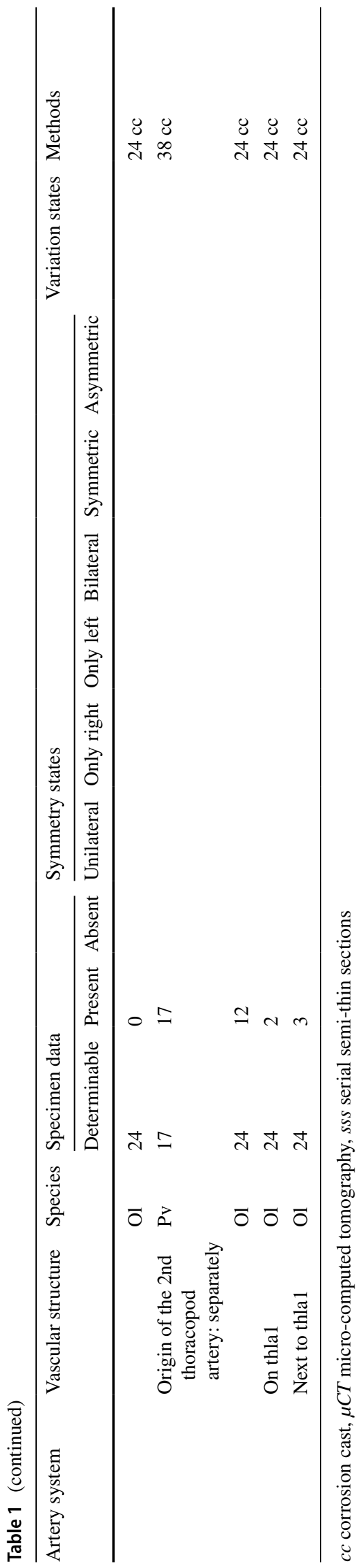

and the genetic uniformity of $P$. virginalis, the intraspecific variability in vascular morphology in the two sexually reproducing crayfish species is most likely to result from the same mechanism.

The numerous regions subject to phenotypic variation in the crayfish vasculature lead to the assumption that mainly the major arteries are genetically determined while most ramifications around other organs are not. The obvious relevance of non-genetic phenotypical variation in vascular morphology bears a significant impact on the interpretation of vascular morphology in terms of putative evolutionary scenarios. Furthermore, such findings on the high prevalence of non-genetic phenotypic variation in morphology need to be considered for evolutionary morphology and the conceptualization of morphological characters in general.

\section{Stochastic developmental variation or phenotypic plasticity due to microenvironments?}

Since phenotypic plasticity and stochastic developmental variation represent complementary aspects of non-genetic phenotypic variation (Furness et al. 2015; Leung et al. 2016), it makes sense to not assess their evolutionary importance as isolated mechanisms but as two aspects of one and the same general phenomenon, i.e. genetic indeterminacy of phenotype development. Although mostly discussed in the light of phenotypic plasticity, suggested evolutionary advantages hold true for non-genetic phenotypic variation in general in due consideration of plasticity and stochastic variation as complementary mechanisms (Simons 2014; Leung et al. 2016). Non-genetic phenotypic variation allows populations to face environmental change in shorter time than genotype evolution could possibly do (Burggren 2018; Angers et al. 2020). Thus, non-genetic phenotypic variation appears evolutionary favorable in variable environments (Hendry 2016; Burggren 2018) with phenotypic plasticity being more frequent in environments with rather predictable change and stochastic developmental variation in unpredictable environments (Furness et al. 2015; Leung et al. 2016).

Stochastic developmental variation produces different phenotypes as result of epigenetic variation (Vogt 2017, 2020; Angers et al. 2020), whereas environmentally induced phenotypes can actually be manifested into the epigenome (Bautista et al. 2020). As transgenerational phenotypic plasticity due to epigenetic inheritance has been shown in several studies (e.g. Holeski et al. 2012; Serobyan and Sommer 2017; Schmid et al. 2018; Bautista and Burggren 2019; Bautista et al. 2020), it is just as plausible to assume such epigenetic inheritance (via inherited methylation patterns, histone modifications, etc.; Holeski et al. 2012; Schmid et al. 2018; Bautista et al. 2020) for phenotypes resulting from stochastic developmental variation. As some phenotypes produced in such a bet-hedging strategy will show a higher 

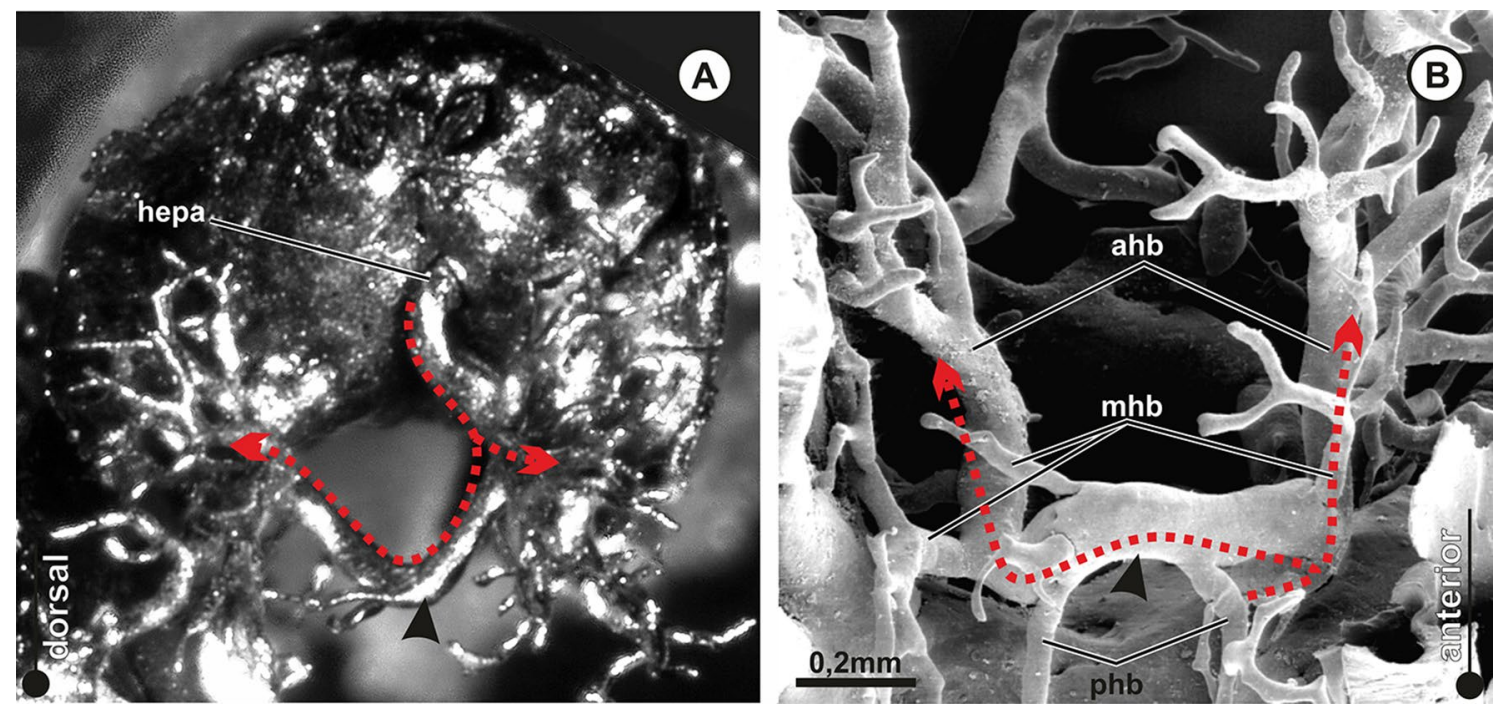

Fig. 6 Unpaired hepatic artery in a specimen of P. virginalis. A Photograph of a corrosion cast in anterior view. B SEM of the same corrosion cast in ventral view. $a h b$ anterior hepatic branch, hepa cardiac hepatic artery, $m h b$ mid hepatic branch; posterior hepatic branch

fitness than others will, frequency of the epialleles will likely be shifted in the next generation. Whether such inherited epialleles become directly incorporated in the genome, however, is yet to be shown on a broader scale. While there are single indications of such direct mechanisms (e.g. Skinner et al. 2015), other indirect effects of epigenetic inheritance on selection and thus evolution are discussed (see Burggren 2016).

A putative selection towards a certain degree of indeterminacy, leading to phenotypic plasticity and stochastic developmental variation, is thus easily assumed, however, appears difficult to be scientifically proven (Via 1993; Pigliucci 2005).

Since mother individuals of the marbled crayfish were kept here under the same conditions, phenotypic plasticity due to the general environmental factors in the tank (e.g. temperature, oxygen saturation, $\mathrm{pH}$, photoperiod) would have to be excluded at first glance. Does this leave stochastic developmental variation (Vogt 2015, 2020) as the only possible explanation for such non-genetic phenotypic variation? Stochastic developmental variation can be ascribed to random epigenetic alterations having an impact on developmental processes (Leung et al. 2016; Angers et al. 2020). The phenotypic variation studied herein might (at least in part) be the result of stochastic developmental variation. However, we think that phenotypic plasticity cannot be excluded completely. While the general conditions in the tank might have been identical for the mother individuals, microenvironments for the developing embryos certainly differ within each clutch. In egg clutches of fishes, position of the individual egg in the egg mass correlates with phenotypic differences (size, body mass, metabolic rate) between the siblings
(Green et al. 2006; Burton et al. 2013). Egg clutches obstruct water flow, which leads to differences in the water chemistry (e.g. oxygen saturation, $\mathrm{pH}$ ) for eggs located within the egg mass (Dhiyebi et al. 2013). These flow-related crowding effects occur already due to mere presence of a single layer of neighboring eggs and are further amplified by their metabolism (Dhiyebi et al. 2013). Such effects may well lead to developmental responses in form of phenotypic plasticity leading to different phenotypes of the offspring as result of different microenvironmental conditions during development. It is thus plausible to assume that the differences, e.g. in oxygen supply, lead to different physiological necessities for the developing organs in the crayfish embryo. Different vascular patterns, a regionally more extensive vasculature or certain anastomoses, as found in this study, might optimize supply for certain developing tissues and organs and might form as result of phenotypic plasticity induced by these microenvironmental conditions.

Characterization of the epigenome alone will also not be able to provide a definitive answer to the question of the origin of the different phenotypes. While phenotypic variation due to stochastic developmental variation follows random epimutations (Angers et al. 2020), phenotypic plasticity, in turn, can lead to manifestation of the environmentally induced phenotypes into the epigenome and even allow for transgenerational epigenetic inheritance of plasticity induced phenotypes (Bautista et al. 2020).

To answer this question, artificial incubation of the eggs with guaranteed identical environmental conditions for each egg needs to be performed. To further clarify, whether the phenotype follows the epigenome or vice versa, methylation patterns, histone modifications etc. would need to be 
compared at the beginning of incubation and after hatching. This, however, appears hardly possible in early embryos without at least altering (in fact rather damaging or sacrificing) them, as epigenetic profiles differ between tissues (Leung et al. 2016) and thus early somatic cells from the embryo would be necessary. Novel strategies to differentially assess phenotypic plasticity and stochastic developmental variation thus need to be developed.

\section{Non-genetic phenotypic variation in evolutionary morphology}

In evolutionary morphology, characters are conceptualized to infer phylogenetic hypotheses and/or reconstruct events of evolutionary transformation (Wirkner and Richter 2010; Richter and Wirkner 2014). Characters represent hypotheses on units of evolution and thus on subjects of selection and adaptation (Wagner 2000, 2014; Richter and Wirkner 2014). Biological individuality and inheritability are considered prerequisites for entities to constitute a character (Wagner 2014). Such morphemes, (i.e. observable physical constituents of organisms; Richter and Wirkner 2014), which are subject to either phenotypic plasticity or stochastic developmental variation are not genetically determined and thus not inheritable in that sense across numerous generations (as opposed to inherited epigenetic variations which generally dissipate over a few generations or even over successive broods of the same generation; Burggren 2015) which means they cannot represent an evolutionary character (Wagner 2000, 2014; Richter and Wirkner 2014; Göpel and Richter 2016). For instance, the branching pattern of the distal portion of the posterior aorta system, cannot be a character in an analysis of crayfish as none of the actually observed patterns is genetically determined and thus inheritable. The fact that a certain morpheme is subject to phenotypic plasticity or stochastic developmental variation, however, can be a character itself in the sense that a lack of genetic determination of a certain morphological pattern in some taxa (as opposed to certain other taxa with genetic determination of said morphological pattern) might be treated analogous to the character state absent in neomorphic characters (Sereno 2007). Non-genetic phenotypic variation must therefore be carefully considered in the context of evolutionary morphology and a sufficient number of individuals need to be investigated in order to conceptualize morphological characters.

\section{Conclusion}

Morphology of the hemolymph vascular system in crayfish shows a significant degree of non-genetic phenotypic variation in terms of varying branching patterns, asymmetries and variably occurring anastomoses. Because of its relevance as supplying organ system in the integral organism, morphology of the circulatory system as well as cardiorespiratory physiology appear worthwhile investigating in terms of both phenotypic plasticity and stochastic developmental variation as response to fast environmental changes (e.g. Leung et al. 2016; Burggren 2018). The marbled crayfish $P$. virginalis has proven suitable as object of study in terms of stochastic developmental variation as well as phenotypic plasticity (Vogt 2020). Future work might include investigation of cause and effects regarding variation in vascular morphology as well as the impact of non-genetic phenotypic variation of cardiorespiratory physiology.

Acknowledgements The authors are grateful to an anonymous crayfish for establishing an own parthenogenetic species to help study of nongenetic phenotypic variation and other phenomena. Two anonymous reviewers provided helpful comments to significantly improve the manuscript, which is gratefully appreaciated. The authors would like to thank Marcus Frank and his team at the EMZ (University of Rostock) for their help with electron microscopy. TG is funded by a Forschungsstipendium from the Deutsche Forschungsgemeinschaft DFG (GO 3341/1-1). CSW received funding from the Deutsche Forschungsgemeinschaft DFG (WI 3334/1-2). The $\mu \mathrm{CT}$ machines at Universität Rostock (DFG INST 264/38-1 FUGG, DFG INST 264/130-1 FUGG) were jointly sponsored by the Deutsche Forschungsgemeinschaft and the Land Mecklenburg-Vorpommern.

Funding Open Access funding enabled and organized by Projekt DEAL.

Open Access This article is licensed under a Creative Commons Attribution 4.0 International License, which permits use, sharing, adaptation, distribution and reproduction in any medium or format, as long as you give appropriate credit to the original author(s) and the source, provide a link to the Creative Commons licence, and indicate if changes were made. The images or other third party material in this article are included in the article's Creative Commons licence, unless indicated otherwise in a credit line to the material. If material is not included in the article's Creative Commons licence and your intended use is not permitted by statutory regulation or exceeds the permitted use, you will need to obtain permission directly from the copyright holder. To view a copy of this licence, visit http://creativecommons.org/licenses/by/4.0/.

\section{References}

Angers B, Perez M, Menicucci T, Leung C (2020) Sources of epigenetic variation and their applications in natural populations. Evol Appl 13:1262-1278

Atlasi Y, Stunnenberg H (2017) The interplay of epigenetic marks during stem cell differentiation and development. Nat Rev Genet 18(11):643-658

Baumann H (1921) Das Gefäßsystem von Astacus fluviatilis (Potamobius astacus L.). Z Wiss Zool 118:246-312

Bautista NM, Burggren WW (2019) Parental stressor exposure simultaneously conveys both adaptive and maladaptive larval phenotypes through epigenetic inheritance in the zebrafish (Danio rerio). J Exp Biol 222(17):jeb208918 
Bautista NM, Crespel A, Crossley J, Padilla P, Burggren WW (2020) Parental transgenerational epigenetic inheritance related to dietary crude oil exposure in Danio rerio. J Exp Biol 223(16):jeb222224

Burggren WW (2015) Dynamics of epigenetic phenomena: intergenerational and intragenerational phenotype "washout." J Exp Biol 218(1):80-87

Burggren WW (2016) Epigenetic inheritance and its role in evolutionary biology: re-evaluation and new perspectives. Biology 5(2):24

Burggren WW (2018) Developmental phenotypic plasticity helps bridge stochastic weather events associated with climate change. J Exp Biol 221:jeb161984

Burton T, Hoogenboom MO, Beevers ND, Armstrong JD, Metcalfe NB (2013) Among-sibling differences in the phenotypes of juvenile fish depend on their location within the egg mass and maternal dominance rank. Proc R Soc B Biol Sci 280(1751):20122441

Chevin LM, Hoffmann AA (2017) Evolution of phenotypic plasticity in extreme environments. Philos Trans R Soc Lond B 372:20160138

Dhiyebi HA, O'Donnell MJ, Wright PA (2013) Water chemistry in the microenvironment of rainbow trout Oncorhynchus mykiss embryos is affected by development, the egg capsule and crowding. J Fish Biol 82(2):444-457

Furness AI, Lee K, Reznick DN (2015) Adaptation in a variable environment: Phenotypic plasticity and bet-hedging during egg diapause and hatching in an annual killifish. Evolution 69(6):1461-1475

Ghalambor CK, McKay JK, Carroll SP, Reznick DN (2007) Adaptive versus non-adaptive phenotypic plasticity and the potential for contemporary adaptation in new environments. Funct Ecol 21:394-407

Göpel T, Richter S (2016) The word is not enough: on morphemes, characters and ontological concepts. Cladistics 32:682-690

Göpel T, Wirkner CS (2015) An "ancient" complexity? Evolutionary morphology of the circulatory system in Xiphosura. Zoology 118:221-238

Green BS, Anthony KR, McCormick MI (2006) Position of egg within a clutch is linked to size at hatching in a demersal tropical fish. J Exp Mar Biol Ecol 329(1):144-152

Gutekunst J, Andriantsoa R, Falckenhayn C, Hanna K, Stein W, Rasamy J, Lyko F (2018) Clonal genome evolution and rapid invasive spread of the marbled crayfish. Nat Ecol Evol 2(3):567-573

Hendry AP (2016) Key questions on the role of phenotypic plasticity in eco-evolutionary dynamics. J Hered 107:25-41

Holeski LM, Jander G, Agrawal AA (2012) Transgenerational defense induction and epigenetic inheritance in plants. Trends Ecol Evol 27(11):618-626

Huckstorf K, Wirkner CS (2019) Comparative morphology of the hemolymph vascular system in mygalomorphs (Araneae; Opisthothelae). J Arachol 47:334-343

Huckstorf K, Michalik P, Ramírez M, Wirkner CS (2015) Evolutionary morphology of the hemolymph vascular system of basal araneomorph spiders (Araneae: Araneomorphae). Arthropod Struct Dev 44(6):609-621

Imafuku M (1993) Observations on the internal asymmetry of the sternal artery and the cheliped asymmetry in selected decapod crustaceans. Crust Res 22:35-43

Keiler J, Richter S, Wirkner CS (2013) Evolutionary morphology of the hemolymph vascular system in Hermit and King Crabs (Crustacea: Decapoda: Anomala). J Morphol 274:759-778

Keiler J, Richter S, Wirkner CS (2015) Evolutionary morphology of the organ systems in squat lobsters and porcelain crabs (Crustacea: Decapoda: Anomala): an insight into carcinization. J Morph 276(1):1-21

Keiler J, Richter S, Wirkner CS (2016) Revealing their innermost secrets: an evolutionary perspective on the disparity of the organ systems in anomuran crabs (Crustacea: Decapoda: Anomura). Contrib Zool 85(4):361-386

Kelly SA, Panhuis TM, Stoehr AM (2012) Phenotypic plasticity: molecular mechanisms and adaptive significance. Compr Physiol 2:1417-1439

Klußmann-Fricke B-J, Prendini L, Wirkner CS (2012) Evolutionary Morphology of the hemolymph vascular system in scorpions: a character analysis. Arthropod Struct Dev 41:545-560

Langerhans RB, DeWitt TJ (2002) Plasticity constrained: over-generalized induction cues cause maladaptive phenotypes. Evol Ecol Res 4:857-870

Leung $C$ et al (2016) Facing environmental predictability with different sources of epigenetic variation. Ecol Evol 6:5234-5245

Lyko F (2017) The marbled crayfish (Decapoda: Cambaridae) represents an independent new species. Zootaxa 4363:544-552

Martin P, Kohlmann K, Scholtz G (2007) The parthenogenetic Marmorkrebs (marbled crayfish) produces genetically uniform offspring. Naturwissenschaften 94:843-846

Martin P, Dorn NJ, Kawai T, van der Heiden C, Scholtz G (2010) The enigmatic Marmorkrebs (marbled crayfish) is the parthenogenetic form of Procambarus fallax (Hagen, 1870). Contrib Zool 79:107-118

Martin P, Thonagel S, Scholtz G (2016) The parthenogenetic Marmorkrebs (Malacostraca: Decapoda: Cambaridae) is a triploid organism. J Zool Syst Evol Res 54:13-21

Pelster B, Burggren WW (2018) Responses to environmental stressors in developing animals: Costs and benefits of phenotypic plasticity. In: Burggren W, Dubansky B (eds) Development and environment. Springer, Cham, pp 97-113

Pigliucci M (2005) Evolution of phenotypic plasticity: where are we going now? Tree 20:481-486

Richter S, Wirkner CS (2014) A research program for evolutionary morphology. J Zool Syst Evol Res 52:338-350

Runge J, Wirkner CS (2016) The hemolymph vascular system in Araneus diadematus with special focus on intraspecific variability in artery systems. J Arachnol 44:153-164

Schmid MW, Heichinger C, Schmid DC, Guthörl D, Gagliardini V, Bruggmann R, Aluri S, Aquino C, Schmid B, Turnbull LA, Grossniklaus U (2018) Contribution of epigenetic variation to adaptation in Arabidopsis. Nat Commun 9(1):1-12

Scholtz G, Braband A, Tolley L, Reimann A, Mittmann B, Lukhaup C, Steuerwald F, Vogt G (2003) Parthenogenesis in an outsider crayfish. Nature 421:806-806

Scholz S, Richter S, Wirkner CS (2018) Constant morphological patterns in the hemolymph vascular system of crayfish (Crustacea, Decapoda). Arthropod Struct Dev 47:248-267

Sereno PC (2007) Logical basis for morphological characters in phylogenetics. Cladistics 23:565-587

Serobyan V, Sommer RJ (2017) Developmental systems of plasticity and trans-generational epigenetic inheritance in nematodes. Curr Opin Gen Dev 45:51-57

Simon JC, Delmotte F, Rispe C, Crease T (2003) Phylogenetic relationships between parthenogens and their sexual relatives: the possible routes to parthenogenesis in animals. Biol J Linn Soc Lond 79:151-163

Simons AM (2014) Playing smart vs. playing safe: the joint expression of phenotypic plasticity and potential bet hedging across and within thermal environments. J Evol Biol 27(6):1047-1056

Skinner MK, Guerrero-Bosagna C, Haque MM (2015) Environmentally induced epigenetic transgenerational inheritance of sperm epimutations promote genetic mutations. Epigenetics 10(8):762-771

Tollrian R (1993) Neckteeth formation in Daphnia pulex as an example of continuous phenotypic plasticity: morphological effects of Chaoborus kairomone concentration and their quantification. $\mathrm{J}$ Plankton Res 15:1309-1318 
Tollrian R (1995) Predator-induced morphological defenses: costs, life history shifts, and maternal effects in Daphnia pulex. Ecology 76:1691-1705

Via S (1993) Adaptive phenotypic plasticity: target or by-product of selection in a variable environment? Am Nat 142:352-365

Vogt G (2008) The marbled crayfish: a new model organism for research on development, epigenetics and evolutionary biology. J Zool 276:1-13

Vogt G (2015) Stochastic developmental variation, an epigenetic source of phenotypic diversity with far-reaching biological consequences. J Biosci 40:159-204

Vogt G (2020) Disentangling the environmentally induced and stochastic developmental components of phenotypic variation. In: Levine H, Jolly MK, Kulkarni P, Nanjundiah V (eds) Phenotypic switching: implications in biology and medicine. Academic Press, San Diego, pp 207-251

Vogt G, Huber M, Thiemann M, van den Boogaart G, Schmitz OJ, Schubart CD (2008) Production of different phenotypes from the same genotype in the same environment by developmental variation. J Exp Zool 211:510-523

Vogt G, Wirkner CS, Richter S (2009) Symmetry variation in the heartdescending artery system of the parthenogenetic marbled crayfish. J Morphol 270:221-226

Vogt G (2017) Facilitation of environmental adaptation and evolution by epigenetic phenotype variation: insights from clonal, invasive, polyploid, and domesticated animals. Environ Epigenet 3:dvx002

Wagner GP (2000) Characters, units and natural kinds: an introduction. In: Wagner GP (ed) The character concept in evolutionary biology. Academic Press, San Diego
Wagner GP (2014) Homology, Genes, and Evolutionary Innovation. Princeton University Press

West-Eberhard MJ (1989) Phenotypic plasticity and the origins of diversity. Annu Rev Ecol Syst 20:249-278

Wirkner CS, Richter S (2004) Improvement of microanatomical research by combining corrosion casts with MicroCT and 3D reconstruction, exemplified in the circulatory organs of the Woodlouse. Microsc Res Technique 64:250-254

Wirkner CS, Richter S (2010) Evolutionary morphology of the circulatory system in Peracarida (Malacostraca; Crustacea). Cladistics 26:143-167

Wirkner CS, Tögel M, Pass G (2013) The arthropod circulatory system. In: Minelli A, Boxshall G, Fusco G (eds) Arthropod biology and evolution. Springer, Berlin, Heidelberg, pp 343-391

Wirkner CS, Göpel T, Runge J, Keiler J, Klussmann-Fricke B-J, Huckstorf K, Scholz S, Mikó I, Yoder ML, Richter S (2017) The first organ-based ontology for arthropods (Ontology of Arthropod Circulatory Systems-OArCS) and its integration into a novel formalization scheme for morphological descriptions. Syst Biol $66: 754-768$

Publisher's Note Springer Nature remains neutral with regard to jurisdictional claims in published maps and institutional affiliations. 